\title{
Automated fastener versus manually tied knots in minimally invasive mitral valve repair: impact on operation time and short- term results
}

\author{
Martin T. R. Grapow ${ }^{1 *}$, Miroslawa Mytsyk ${ }^{1}$, Jens Fass ${ }^{2}$, Patrick Etter ${ }^{1}$, Peter Matt ${ }^{1}$, Friedrich S. Eckstein ${ }^{1}$
} and Oliver T. Reuthebuch ${ }^{1}$

\begin{abstract}
Background: This study compares the influence of two different annuloplasty attachment suture applications, namely the use of an automated fastener versus manually tied knots using a traditional knot pusher, on total operation time, on cardiopulmonary-bypass time and on cross-clamp time, and on short-term outcome.

Methods: Sixty patients underwent isolated minimally invasive mitral valve repair in Carpentier Type-II mitral disease with implantation of an annuloplasty ring in combination with correction of the prolapsing leaflet using artificial chords. The first 30 patients after implementation of a novel automated fastener were compared with the last 30 patients corrected with a traditional knot pusher. No significant differences with regard to demographic data (age, gender, NYHA class, ejection fraction, BMI, cardiovascular risk factors) between the two groups were found. All patients received isolated mitral valve repair in the first run. Bretschneider HTK was used for cardioplegic cardiac arrest in all patients.

Results: Transesophageal and transthoracic echocardiography at the end of operation and at discharge revealed no $(n=25)$, trace $(n=28)$ or mild $(n=7)$ residual regurgitation with no evidence of ring dehiscence and without any significant clinical differences between the groups. Cross-clamp, cardiopulmonary-bypass and total- operation time were significantly reduced in the automated fastener group compared to the group using a traditional knot pusher $(87.1 \pm 17.9$ vs. $101.3 \pm 17.8 ; p<0.01,138.1 \pm 25.6$ vs. $152.7 \pm 24.9 ; p<0.05$, and $203.9 \pm 31.02$ vs. $223.8 \pm 29.01 ; p<0.01$, respectively).

Conclusion: Our short-term results indicate a safe, reliable and fast application of the novel automated fastener device in combination with significant time savings in cardioplegic arrest and cardiopulmonary bypass.
\end{abstract}

Keywords: Cor-Knot, Automated fastener, Minimally invasive mitral valve repair, Manually tied knots, Cross-clamp

\section{Background}

Mitral valve dysfunction is the second-most common clinically significant form of valvular defect in adults [1]. During the last 15 years, minimally invasive mitral valve surgery (MIMVS) using the right anterolateral mini-thoracotomy access has emerged as an accepted approach for the management of mitral valve disease alone or in combination with tricuspid

\footnotetext{
* Correspondence: Martin.grapow@usb.ch

${ }^{1}$ Department of Cardiac Surgery, University Hospital Basel, Spitalstrasse 21, $\mathrm{CH}-4031$, Basel, Switzerland

Full list of author information is available at the end of the article
}

valve disease, defects of the atrial septum or atrial rhythm disorders with equivalent results [2]. This strategy was developed to decrease surgical trauma by minimizing the size of incisions. It permits excellent exposure of the mitral valve, thereby avoiding conventional full sternotomy. This results in better visualization of the valve for the surgeon and even for all other participants in the OR due to transmission via monitor, faster mobilization of the patient, faster healing, less pain, less infection and better cosmetics. One constraint of minimally invasive surgery is the 
need for remote knot tying, which is typically accomplished with the use of a knot pusher [3]. The challenge of video-assisted knot tying, at disadvantageous angles, has long been recognized as one important drawback in the advancement of minimally invasive surgery. The main problem is that knot tying using endoscopic or robotic-assisted tools is time-consuming, leading to increased operation times $[4,5]$. Operating time is especially critical for cardiac procedures involving cardiopulmonary bypass (CPB). Increased CPB times lead to higher risks of multiple organ dysfunction syndrome [6], and therefore adverse outcomes [7]. Patient mortality rates increase with the cross-clamp time, especially in older patients and during emergency procedures. Furthermore, older patients and otherwise "unhealthy" patients represent the majority of candidates requiring surgical procedures. The Cor- Knot automated fastener (LSI SOLUTIONS, Victor, NY, USA) was introduced in our hospital in November 2013. The aim of our retrospective study is to investigate the influence of manual and automated knot-tying systems on cross-clamp, $\mathrm{CPB}$ and total operation time in one defined operative procedure: isolated minimally invasive mitral valve repair via a right anterolateral, endoscopically assisted thoracotomy with femoral cannulation for the cardiopulmonary bypass (CPB) in Carpentier Type-II pathology.

\section{Methods \\ Patients}

We started our minimally invasive mitral valve program at University Hospital Basel in 2010, reaching a volume of $60-70$ patients per year. From a total of 69 patients who received mitral valve repair in a minimally invasive setting from May 2013 until June 2014, 60 patients we evaluated retrospectively. These patients underwent isolated minimally invasive mitral valve repair in Carpentier Type-II mitral disease, all of them having an annuloplasty ring implanted in combination with correction of the prolapsing leaflet using artificial chords (GoreTex CV4). Repair was performed in the first run without the need for any further correction. Thirty patients after implementation of a novel automated fastener (November 2013June 2014) were compared with 30 patients corrected with a traditional knot pusher (May 2013- October 2013). To increase homogeneity in both groups the following 9 patients were excluded: Patients, who underwent combination surgery with tricuspid valve repair or atrial septal defect closure $(n=3)$, Patients just receiving a ring- annuloplasty $(n=5)$ and one patient who needed a second cross-clamp period for a suboptimal result in the first run. No significant differences regarding demographic data (age, gender, NYHA class, ejection fraction, BMI, cardiovascular risk factors) between the two groups were found (Table 1). Three patients presented with an anterior leaflet prolapse in the knot-pusher group compared to 4 patients in the Cor-knot group. All other patients had the prolapsing segment located at the posterior leaflet. The study was approved by our local ethics comity.

\section{Operative technique}

Minimally invasive mitral valve surgery is performed through a right mini-thoracotomy using standardized methods. Briefly, standard monitoring lines are inserted and the patient is intubated with a doublelumen endotracheal tube. Access to the thoracic cavity is achieved via a 4-6 cm incision in the inframammary groove that is carried out through the third or fourth intercostal space. A soft tissue retractor is used to limit rib spreading. $\mathrm{CPB}$ is set up routinely with femoral vessel cannulation. The opened pericardium is retracted toward the right. Cardiopulmonary bypass is initiated with intrathoracic clamping of the aorta (Glauber-Aortic-Clamp), and the heart is arrested with antegrade infusion of crystalloid cardioplegia (Bretschneider Solution, Custodiol). After cardiac arrest, the left atrium is entered along the interatrial groove. Mitral valve repair is performed using standardized techniques (Annuloplasty ring, Neo Chordae, Gore-Tex Loops). Carbon dioxide field insufflation and standardized transesophageal echocardiography-guided de-airing techniques are routinely used in our clinic. After atrial closure and weaning from $\mathrm{CPB}$, a complete postoperative transesophageal echocardiographic study is recorded. Post-

Table 1 Patients' characteristics

\begin{tabular}{llll}
\hline Characteristic & Manual & Cor-Knot & $p$ \\
\hline No. of patients & 30 & 30 & n.s. \\
Age & $56.13 \pm 16.11$ & $58.8 \pm 13.84$ & n.s. \\
Male & $24(80 \%)$ & $17(57 \%)$ & n.s. \\
Female & $6(20 \%)$ & $13(43 \%)$ & n.s. \\
BMl & $25.96 \pm 3.58$ & $24.95 \pm 6.38$ & n.s. \\
Height & $176 \pm 9.1$ & $174 \pm 10.3$ & n.s. \\
Weight & $80.5 \pm 12.1$ & $76.5 \pm 20.4$ & n.s. \\
Diabetes & $1(3.3 \%)$ & $1(3.3 \%)$ & n.s. \\
Smokers & $4(13.3 \%)$ & $2(6.6 \%)$ & n.s. \\
Dyslipidemia & $6(19.9 \%)$ & $9(29.7 \%)$ & n.s. \\
Hypertension & $15(49.5 \%)$ & $22(72.6 \%)$ & n.s. \\
Anterior leaflet prolapse & $3(10 \%)$ & $22(72.6 \%)$ & n.s. \\
Anterior leaflet prolapse & $3(10 \%)$ & $4(13.3 \%)$ & n.s. \\
Ejection fraction & $60.73 \pm 9.20$ & $58.8 \pm 8.63$ & n.s. \\
Euroscore 2 & $1.03 \pm 0.64$ & $1.21 \pm 0.85$ & n.s. \\
\hline
\end{tabular}


repair echocardiographic data are obtained after closure of the incision.

\section{The automated fastener}

The Cor-Knot device is indicated for use in the approximation of soft tissue and prosthetic materials, used in conjunction with either specified 2-0 polyester or 2-0 polypropylene or 3-0 polypropylene suture and a CorKnot quick load. The pusher can be released only under tension (Fig. 1). The fastener is made of titanium.

\section{Statistical analysis}

Continuous data are presented as mean with standard deviation (SD). Categorical data are presented as absolute numbers and percentages. Differences between groups are calculated with the Student's $t$-test. Overall significance is attained with a $p<0.05$. The analysis was performed with the use of Microsoft Office Excel (Microsoft, Redmond, WA, USA).

\section{Results}

Transesophageal and transthoracic echocardiography at the end of operation and at discharge revealed no $(n=25)$, trace $(n=28)$ or mild $(n=7)$ residual regurgitation with no evidence of ring dehiscence and without any significant differences between the two groups. Furthermore, no differences were detected in ICU stay, total length of hospital stay or the need for blood transfusion (Table 2). There were no myocardial infarction, stroke, limb ischemia or respiratory problems noted in these patients. All patients survived the procedure and were discharged from hospital.

The amount of sutures needed for ring fixation were similar in both groups, either in total or as analyzed per patient. Cross-clamp time, CPB time and operation time were significantly reduced in the automated fastener group compared to the group using a traditional knot pusher $(87.1 \pm 17.9$ vs. $101.3 \pm 17.8 ; p<0.01,138.1 \pm 25.6$ vs. $152.7 \pm 24.9 ; p<0.05$, and $203.9 \pm 31.02$ vs. $223.8 \pm$ 29.01; $p<0.01$, respectively). (Table 3 , Figs. 2,3 and 4.)

\section{Discussion}

The apparent advantages of minimally invasive mitral surgery through right mini- thoracotomy are considered to be a very small wound surface area, rapid recovery with shorter ventilation time, shorter hospital stay and less blood transfusion, as well as reduced postoperative pain, and better cosmetic result [8]. Furthermore, perfect visualization of the valve in high definition and live transmission via multiple monitors guarantee access for all participants in the OR. The valve site is not only seen by one single surgeon; in fact, more eyes can follow the operation with a possible impact on safety - even teaching is much more feasible. The drawbacks of this technique are perfusion-related complications (e.g. stroke, vascular damage and limb ischemia), which tend to occur more frequently in minimally invasive technique than with the standard technique [9]. In addition, valve repair through a small thoracotomy is technically demanding, leading to prolonged cross-clamp, $\mathrm{CPB}$ and operation times with a potential negative impact on outcome.

The results of our study show that with the introduction of the Cor-Knot device (Fig. 3 in our hospital (November 2013), cross-clamp time, CPB time and total operation time were significantly reduced compared to our control group (Fig. 4). Time savings varied from 14 (cross-clamping) up to $20 \mathrm{~min}$ (operation). Usually 15 to 20 sutures are used for fixation of an annuloplasty ring in our clinic. The time saving in our study is consistent with the results
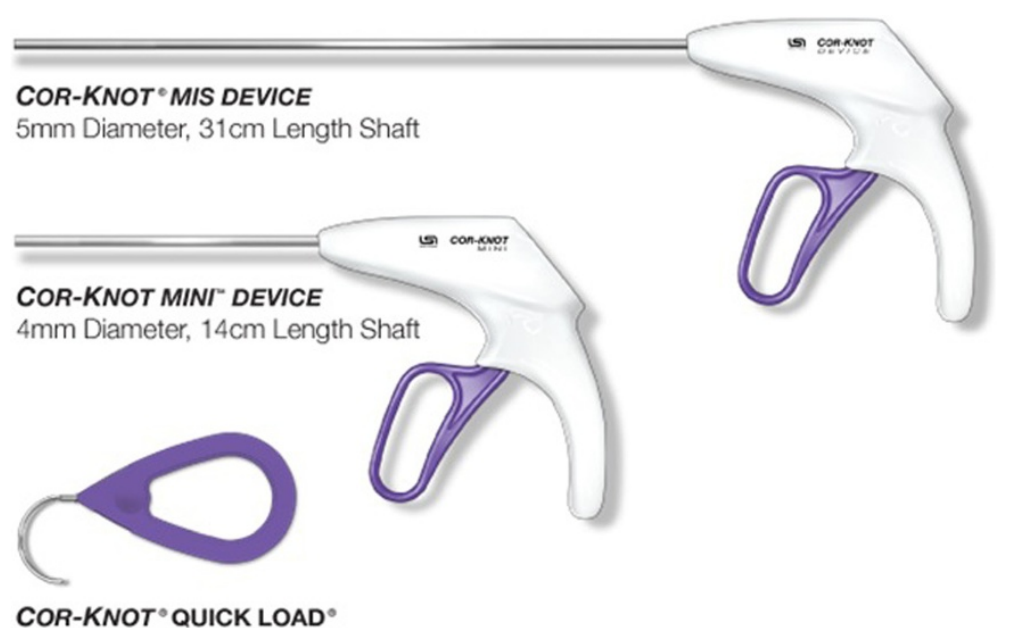

Fig. 1 The Cor-Knot.Device 
Table 2 Outcome

\begin{tabular}{llll}
\hline Characteristic & Manual & Cor-Knot & $p$ \\
\hline Intensive care Unit (days) & $2.5 \pm 2.33$ & $2.2 \pm 1.65$ & n.s. \\
Hospitalization duration (days) & $10.0 \pm 5.42$ & $11.6 \pm 12.2$ & n.s \\
Blood products per patient & $0.7 \pm 1.68$ & $0.55 \pm 1.12$ & n.s. \\
\hline
\end{tabular}

obtained by Lee et al. [10], in which the Cor- Knot device was compared with hand-tied knots using a knot pusher in an ex vivo porcine minimally invasive simulation model. A significant reduction in time of almost one minute was obtained for the Cor-Knot device for one knot (12.4 vs 71.1 seconds per knot, $p=0.001$ ) as compared with the manually tied knot using the traditional knot pusher. Another important feature of the Cor-Knot device is the release of the fastener, which is only possible under tension, thereby guaranteeing the requested tissue attachment. Undesirable loose knots, which can occur by placing hand-tied knots with a knot pusher with additional time loss, are eliminated.

Saving time during cross-clamping provides the surgeon with more flexibility in case of a suboptimal mitral repair result and the need for a second or even a third run of cross-clamping with additional readjustment procedures. Increased cross-clamp and CPB time have been shown to be independent predictors of morbidity and mortality in cardiac surgery $[11,12]$.

In our study, no differences in clinical outcome, i.e. minor or major adverse events, were noted. All patients

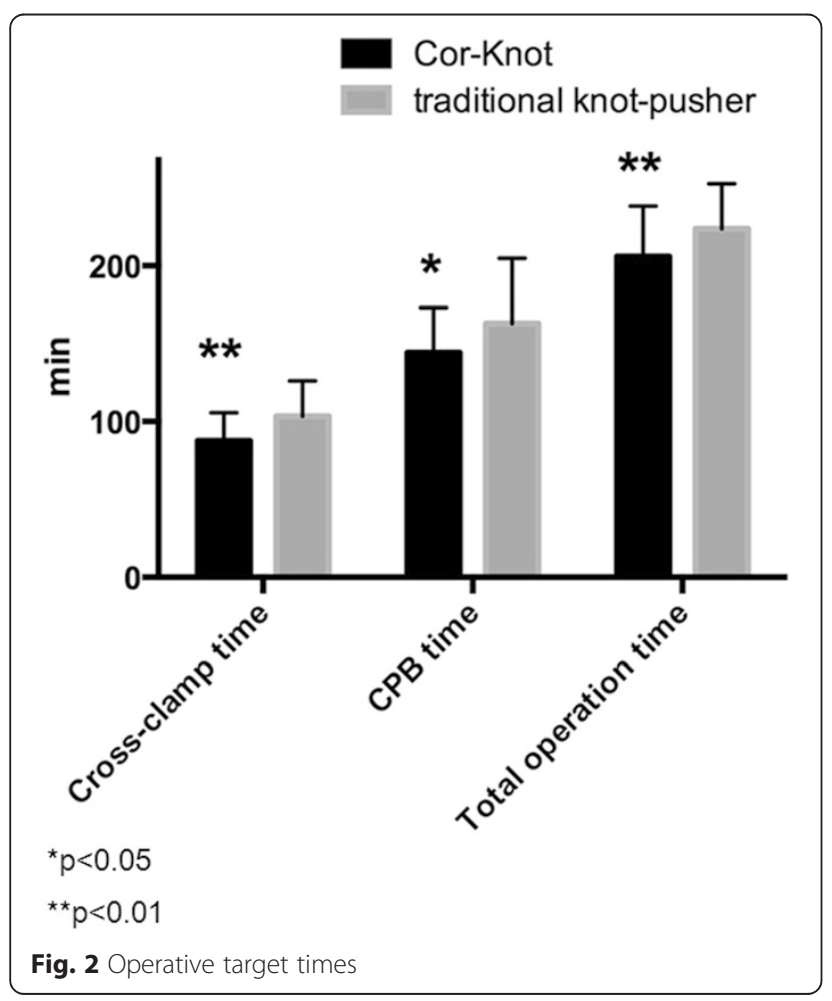

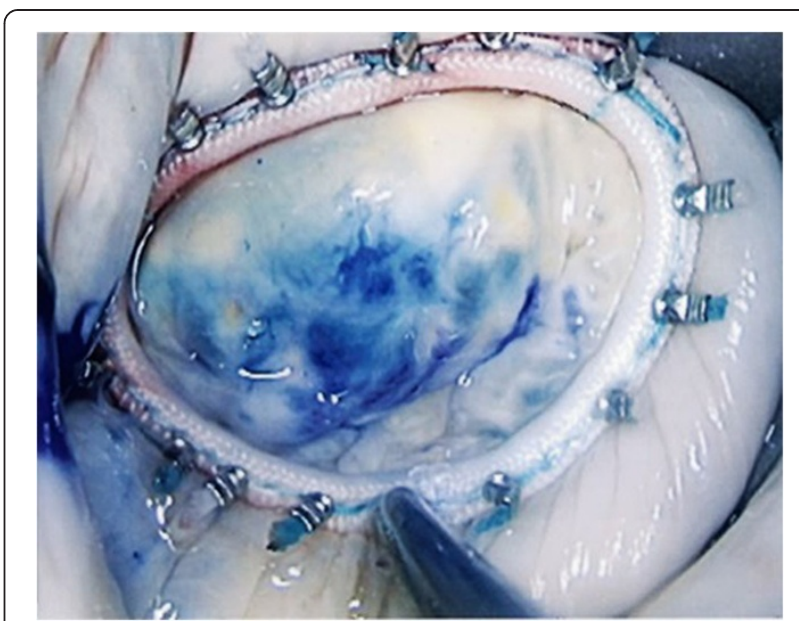

Fig. 3 Mitral valve after repair and ring annuloplasty with Cor-Knot fixation

safely left our hospital for cardiac rehabilitation programs. All underwent repair of the mitral valve in the first run (one cross-clamping period) with the anticipated good operative results and therefore no significant differences in outcome are to be expected, especially given the lack of power with only 60 included patients.

Another important issue is economics: The Cor-Knot device with the application of 20 fasteners costs around 1000 Swiss Francs, which has to be compared with the operative time saving of $15-20 \mathrm{~min}$. In Basel, one minute in the cardiac surgery OR costs around 80 Swiss Francs, which means a reduction in OR costs of between 1200 and 1600 Swiss Francs and a profit of 200-600 Swiss Francs.

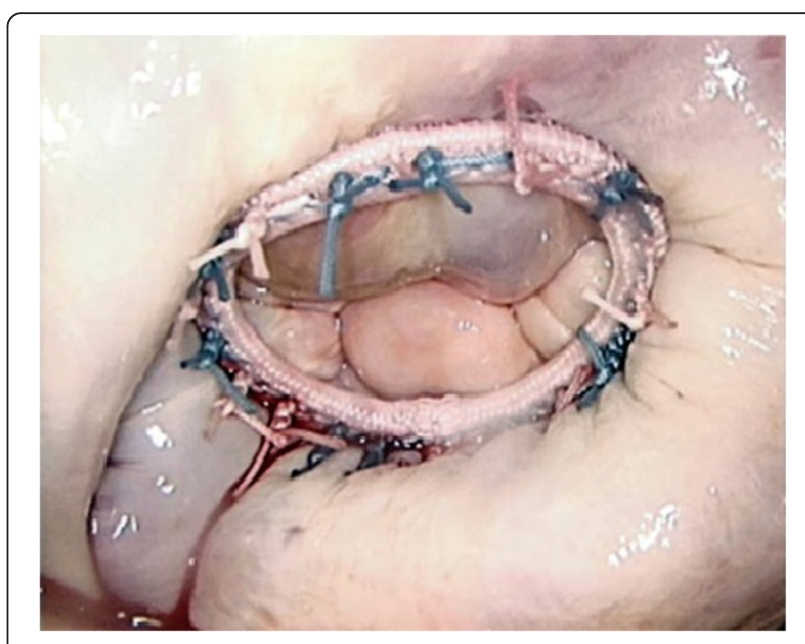

Fig. 4 Mitral valve after repair with traditional ring fixation using a knot pusher 
Table 3 Intraoperative data

\begin{tabular}{llll}
\hline Characteristic & Cor-Knot & Manual & $p$ \\
\hline No. of knots, total & 455 & 442 & n.s. \\
No. of knots, per patient & $15.1 \pm 1.86$ & $14.7 \pm 1.9$ & n.s. \\
Cross-clamp time & $87.1 \pm 17.9$ & $101.3 \pm 17.8$ & $<0.01$ \\
ECC time & $138.1 \pm 25.6$ & $152.7 \pm 24.9$ & $<0.05$ \\
Operation time & $203.9 \pm 31.02$ & $223.8 \pm 29.01$ & $<0.01$ \\
\hline
\end{tabular}

\section{Conclusion}

Our short-term results indicate a safe, reliable and fast application of the novel automated fastener device in combination with significant time savings in cardioplegic arrest and cardiopulmonary bypass.

\section{Competing interests}

The authors declare that they have no competing interests.

\section{Authors' contributions}

MG performed MIMVS, planned and supervised study, carried out statistical analysis as well as manuscript preparation. MM carried out data collection and manuscript preparation. PE organized data collection and update of literature. JF performed perioperative echocardiography. PM revised the manuscript. FE performed MIMVS and revised manuscript. OR performed MIMVS, planned and supervised study, carried out statistical analysis as well as manuscript preparation.

\section{Author details}

${ }^{1}$ Department of Cardiac Surgery, University Hospital Basel, Spitalstrasse 21, $\mathrm{CH}-4031$, Basel, Switzerland. ${ }^{2}$ Department of Anesthesia, University Hospital Basel, Spitalstrasse 21, CH-4031, Basel, Switzerland.

Received: 29 May 2015 Accepted: 26 October 2015

Published online: 03 November 2015

\section{References}

1. Madesis A, Tsakiridis K, Zarogoulidis P, Katsikogiannis N, Machairiotis N, Kougioumtzi I. Review of mitral valve insufficiency: repair or replacement. J Thorac Dis. 2014;6 Suppl 1:S39-51.

2. Khaled D. Algarnia, Rakesh M. Suria, Hartzell Schaff. Minimally invasive mitral valve surgery: does it make a difference? Trends Cardiovasc Med. 2015;25:456-65

3. Jernigan SR1, Chanoit G, Veeramani A, Owen SB, Hilliard M, Cormier D, et al. A laparoscopic knot-tying device for minimally invasive cardiac surgery. Eur J Cardiothorac Surg. 2010;37:626-30.

4. Dogan S, Aybek T, Risteski PS, Detho F, Rapp A, Wimmer-Greinecker G, et al. Minimally invasive port access versus conventional mitral valve surgery: prospective randomized study. Ann Thorac Surg. 2005;79:492-8.

5. Raanani E1, Spiegelstein D, Sternik L, Preisman S, Moshkovitz Y, Smolinsky AK, et al. Quality of mitral valve repair: median sternotomy versus portaccess approach. J Thorac Cardiovasc Surg. 2010;140:86-90.

6. Sablotzki A, Friedrich I, Muhling J, Dehne MG, Spillner J, Silber RE, et al. The systemic inflammatory response syndrome following cardiac surgery: different expression of proinflammatory cytokines and procalcitonin in patients with and without multiorgan dysfunctions. Perfusion. 2002;17:103-9.

7. Trescher K, Gleiss A, Boxleitner M, Dietl W, Kassal H, Holzinger C et al. Shortterm clinical outcomes between intermittent cold versus intermittent warm blood cardioplegia in 2200 adult cardiac surgery patients. J Cardiovasc Surg (Torino). 2015, [Epub ahead of print].

8. Ito T. Minimally invasive mitral valve surgery through right minithoracotomy: recommendations for good exposure, stable cardiopulmonary bypass, and secure myocardial protection. Gen Thorac Cardiovasc Surg. 2015;63:371-8.

9. Ad N, Holmes SD, Shuman DJ, Pritchard G. Minimally invasive mitral valve surgery without aortic cross-clamping and with femoral cannulation is not associated with increased risk of stroke compared with traditional mitral valve surgery: a propensity score-matched analysis. Eur J Cardiothorac Surg. 2015; [Epub ahead of print].

10. Lee CY, Sauer JS, Gorea HR, Martellaro AJ, Knight PA. Comparison of strength, consistency, and speed of COR-KNOT versus manually hand-tied knots in an ex vivo minimally invasive model. Innovations (Phila). 2014;9:111-6.

11. Salis S, Mazzanti W, Merli G, Salvi L, Tedesco CC, Veglia F, et al. Cardiopulmonary bypass duration is an independent predictor of morbidity and mortality after cardiac surgery. J Cardiothorac Vasc Anesth. 2008;22:814-22.

12. Nissinen J, Biancari F, Wistbacka JO, Peltola T, Loponen P, Tarkiainen P, et al. Safe time limits of aortic cross-clamping and cardiopulmonary bypass in adult cardiac surgery. Perfusion. 2009;24:297-305.

\section{Submit your next manuscript to BioMed Central and take full advantage of:}

- Convenient online submission

- Thorough peer review

- No space constraints or color figure charges

- Immediate publication on acceptance

- Inclusion in PubMed, CAS, Scopus and Google Scholar

- Research which is freely available for redistribution

Submit your manuscript at www.biomedcentral.com/submit 